\title{
MODELING AND PARAMETER ESTIMATION FOR AN IMPERFECTLY CLAMPED PLATE *
}

\author{
H.T. Banks \\ Center for Research in Scientific Computation \\ North Carolina State University \\ Raleigh, NC 27695 \\ R.C. Smith \\ Yun Wang \\ Department of Mathematics \\ Iowa State University \\ Ames, IA 50011 \\ Mathematical Products Division \\ Armstrong Laboratory \\ Brooks AFB, TX 78235
}

\begin{abstract}
In this paper, the modeling of boundary conditions for an imperfectly clamped circular plate is discussed. This model is derived under the assumption that there is some variation in both the boundary displacement and slope when the plate oscillates. To account for this oscillation, boundary moment terms, involving parameters to be estimated through fit-todata techniques, are included in the model. The well-posedness of the model is demonstrated and appropriate approximation techniques are outlined. Finally, under natural assumptions for this model and approximation scheme, results leading to parameter convergence are given.
\end{abstract}

\section{Introduction}

An important consideration in the modeling of structural and structural acoustic systems involves the determination of appropriate boundary conditions for the vibrating structure. In many applications, the clamped nature of the structure leads to the use of clamped or fixed boundary conditions, in which case, it is assumed that zero displacements and slopes are maintained at the boundaries. For example, this can be an appropriate assumption when using shell equations to model a fuselage, plate

\footnotetext{
*The research of H.T.B. and Y.W. was supported in part by the Air Force Office of Scientific Research under grant AFOSR-F49620-93-1-0198. This research was also supported by the National Aeronautics and Space Administration under NASA Contract Numbers NAS1-18605 and NAS1-19480 while the authors were visiting scientists at the Institute for Computer Applications in Science and Engineering (ICASE), NASA Langley Research Center, Hampton, VA 23681. Additional support was also proved in part under NASA grant NAG-1-1600.
} 


\section{H.T. BANKS, R.C. SMITH AND YUN WANG}

equations to model panels in a transformer or beam equations to model a helicopter blade. In the first case, the experimental shell structures are often supported by heavy clamps at the ends, thus leading to the use of fixed boundary conditions. In a similar manner, the bonding of panels to an underlying substructure or the attachment of the blades to a central hub lead to models which involve fixed boundary conditions.

In both experiments and physical applications, however, it is impossible to maintain truly fixed or clamped boundary conditions. This can be due to physical properties of the system being modeled, limitations in the clamping mechanisms, or material properties which prevent perfect clamps (for example, slipping will occur if the material in the structure being clamped is significantly softer than the clamps). One manner in which the energy loss due to movement in the boundary conditions is manifested is through a lowering of the natural frequencies for the structure or structural acoustic system. This shift in frequencies can range from levels of $1-2 \%$ in well-clamped systems to $20-30 \%$ in loosely clamped systems [11]. These loosely-clamped systems still support a moment, however, so that simplysupported or pinned boundary conditions (zero displacement and zero moment) are not appropriate.

To determine when the assumption of fixed boundary conditions (zero displacement and slope) yields a sufficiently accurate model, and to provide a means of accounting for the energy loss when this assumption is not appropriate, it is necessary to provide a more comprehensive model for imperfectly clamped boundary conditions.

In this paper, we discuss a model for imperfectly clamped boundary conditions that is derived under the assumption that there is some variance in both the displacement and slope. To account for this variance, boundary moment expressions involving four undetermined parameters are employed. In applications, these parameters, as well as other physical parameters are determined through fit-to-data techniques. The modeling of these boundary conditions in the context of a loosely-clamped circular plate, to which piezoceramic patches are bonded, is presented in the second section of this paper. The presentation includes both the strong and weak forms of the equations of motion as well as a discussion pertaining to when each is useful. In Section 3, the modeling equations are posed in terms of sesquilinear forms and well-posedness issues are discussed. State approximation is addressed in Section 4 and parameter estimation is discussed in Section 5. Finally, we point out that while this model for the boundary conditions is presented in the context of a clamped circular plate, analogous expressions follow for structures involving beams and shells. 


\section{MODEL FOR AN IMPERFECTLY CLAMPED PLATE}

\section{Modeling Equations}

The derivation which follows will be for a circular plate although analogous arguments can be used for plates having other geometries, as well as shells and beams. We will consider a plate of radius $a$ and thickness $h$ as depicted in Figure 2.1. The region occupied by the unstrained neutral surface of the plate will be denoted by $\Gamma_{0}$. We also consider the possibility of piezoceramic patches being bonded to the plate in either axisymmetric or general configurations. The piecewise continuous density, Young's modulus, Poisson ratio, and damping coefficient for the combined structure are denoted $\rho, E, \nu$ and $c_{D}$, respectively.
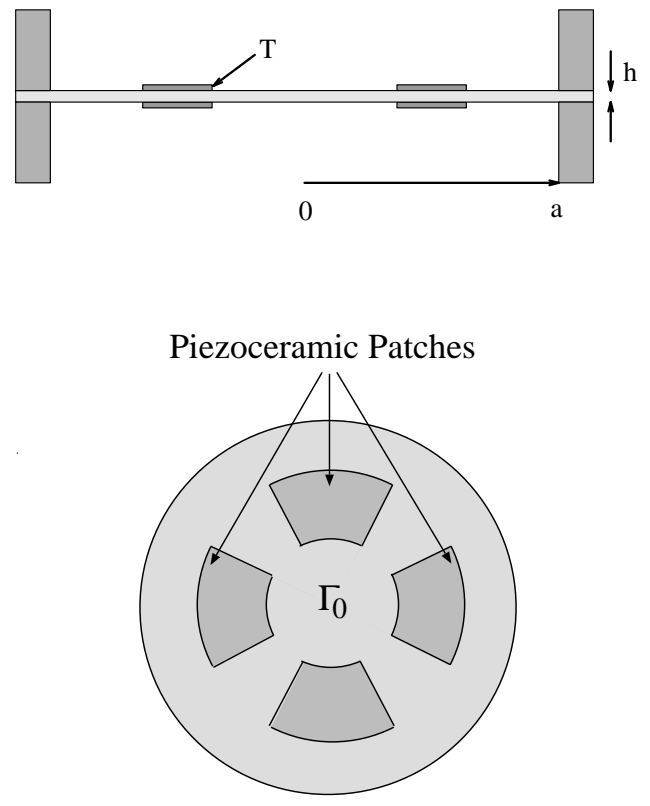

Figure 2.1: A thin circular plate with piezoceramic patches bonded in pairs to its surface.

\subsection{Plate Equations}

To specify moments and forces acting on various regions of the plate, we let $\mathcal{M}_{r}$ and $\mathcal{M}_{\theta}$ denote general moment resultants with respect to $\theta$ and $r$, respectively, and take $Q_{r}$ and $Q_{\theta}$ as corresponding shear resultants. The 


\section{H.T. BANKS, R.C. SMITH AND YUN WANG}

twisting moments are denoted by $\mathcal{M}_{r \theta}$ and $\mathcal{M}_{\theta r}$. As detailed for the axisymmetric case in [14] and the general nonaxisymmetric problem in [10] and (A1), (A3) and (A4) of [5], moment and force balancing for the circular plate then yields

$$
\begin{aligned}
& Q_{r}=\frac{1}{r} \mathcal{M}_{r}+\frac{\partial \mathcal{M}_{r}}{\partial r}-\frac{1}{r} \mathcal{M}_{\theta}+\frac{1}{r} \frac{\partial \mathcal{M}_{\theta r}}{\partial \theta} \\
& Q_{\theta}=\frac{2}{r} \mathcal{M}_{r \theta}+\frac{\partial \mathcal{M}_{r \theta}}{\partial r}+\frac{1}{r} \frac{\partial \mathcal{M}_{\theta}}{\partial \theta}
\end{aligned}
$$

and

$$
Q_{r}+\frac{\partial Q_{r}}{\partial r} r+\frac{\partial Q_{\theta}}{\partial \theta}=-r f+r \rho h \frac{\partial^{2} w}{\partial t^{2}}
$$

where $f$ denotes a surface load on the plate and $w$ is the transverse plate displacement. The sense of the internal moments and forces is illustrated in Figure 2.2. Elimination of shear terms then yields

$$
\begin{gathered}
\rho h \frac{\partial^{2} w}{\partial t^{2}}-\frac{\partial^{2} \mathcal{M}_{r}}{\partial r^{2}}-\frac{2}{r} \frac{\partial \mathcal{M}_{r}}{\partial r}+\frac{1}{r} \frac{\partial \mathcal{M}_{\theta}}{\partial r}-\frac{2}{r} \frac{\partial^{2} \mathcal{M}_{r \theta}}{\partial r \partial \theta} \\
-\frac{2}{r^{2}} \frac{\partial \mathcal{M}_{r \theta}}{\partial \theta}-\frac{1}{r^{2}} \frac{\partial^{2} \mathcal{M}_{\theta}}{\partial \theta^{2}}=f(t, r, \theta) .
\end{gathered}
$$

The general moments are given by

$$
\begin{aligned}
\mathcal{M}_{r} & =M_{r}-\left(M_{r}\right)_{p e} \\
\mathcal{M}_{\theta} & =M_{\theta}-\left(M_{\theta}\right)_{p e} \\
\mathcal{M}_{r \theta} & =M_{r \theta}
\end{aligned}
$$

where

$$
\begin{aligned}
& M_{r}=-D\left(\frac{\partial^{2} w}{\partial r^{2}}+\frac{\nu}{r} \frac{\partial w}{\partial r}+\frac{\nu}{r^{2}} \frac{\partial^{2} w}{\partial \theta^{2}}\right)-c_{D} \frac{\partial}{\partial t}\left(\frac{\partial^{2} w}{\partial r^{2}}+\frac{\nu}{r} \frac{\partial w}{\partial r}+\frac{\nu}{r^{2}} \frac{\partial^{2} w}{\partial \theta^{2}}\right) \\
& M_{\theta}=-D\left(\frac{1}{r} \frac{\partial w}{\partial r}+\frac{1}{r^{2}} \frac{\partial^{2} w}{\partial \theta^{2}}+\nu \frac{\partial^{2} w}{\partial r^{2}}\right)-c_{D} \frac{\partial}{\partial t}\left(\frac{1}{r} \frac{\partial w}{\partial r}+\frac{1}{r^{2}} \frac{\partial^{2} w}{\partial \theta^{2}}+\nu \frac{\partial^{2} w}{\partial r^{2}}\right) \\
& M_{r \theta}=-D(1-\nu)\left(\frac{1}{r} \frac{\partial^{2} w}{\partial r \partial \theta}-\frac{1}{r^{2}} \frac{\partial w}{\partial \theta}\right)-c_{D}(1-\nu) \frac{\partial}{\partial t}\left(\frac{1}{r} \frac{\partial^{2} w}{\partial r \partial \theta}-\frac{1}{r^{2}} \frac{\partial w}{\partial \theta}\right)
\end{aligned}
$$

are the internal plate moments (see $[7,10]$ for derivations of these moment expressions). As detailed in [7], the density $\rho$ in (2.3) and parameters $D=\frac{E h^{3}}{12\left(1-\nu^{2}\right)}, \nu$ and $c_{D}$ in the moment expressions are discontinuous due 


\section{MODEL FOR AN IMPERFECTLY CLAMPED PLATE}

the geometrical and material changes resulting from the bonding of the patches to the plate. The external moments

$$
\left(M_{r}\right)_{p e}=\left(M_{\theta}\right)_{p e}=\sum_{i=1}^{s} \mathcal{K}_{i}^{B} u_{i}(t) \chi_{i}(r, \theta)
$$

are generated by $s$ pairs of patches in response to applied voltages. Here $\chi_{i}(r, \theta)$ denotes the characteristic function which has a value of 1 in the region covered by the $i^{t h}$ patch and is 0 elsewhere. Moreover, $u_{i}(t)$ is the voltage into the $i^{t h}$ patch and $\mathcal{K}_{i}^{B}$ is a parameter which depends on the geometry, piezoceramic material properties and piezoelectric strain constants (see [7] for details). We point out that the piezoceramic material parameters $\mathcal{K}_{i}^{B}, i=1, \cdots, s$ as well as the plate parameters $\rho, D, c_{D}$ and $\nu$ should be considered as unknown and in applications must be estimated using data fitting techniques. The piecewise nature of the material parameters and input moments is one motivation for approximating the problem in a weak or variational form.

We also point out that in general, the patches are not bonded near the edge of the plate. Hence $\mathcal{M}_{r}=M_{r}$ and $\mathcal{M}_{\theta}=M_{\theta}$ at $r=a$ since there are no contributing external moments.

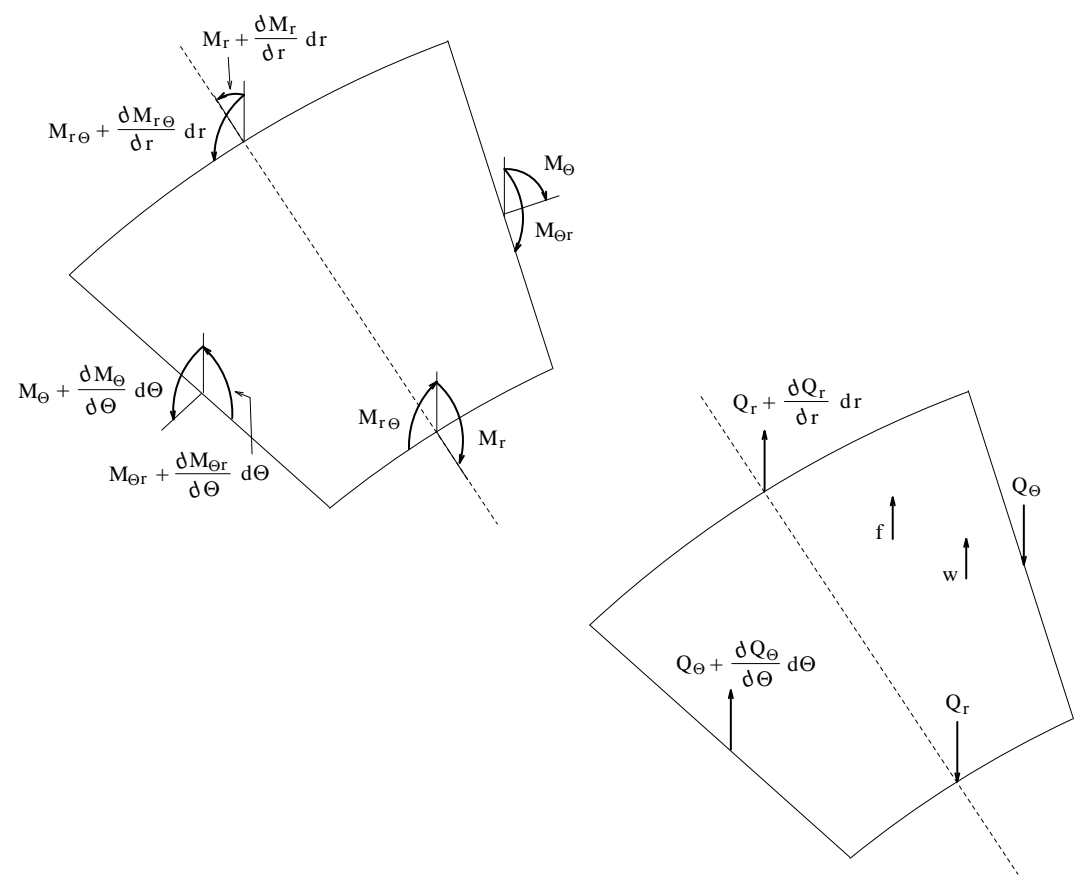

Figure 2.2: The internal moments and forces for the circular plate. 


\section{H.T. BANKS, R.C. SMITH AND YUN WANG}

\subsection{Fixed Boundary Conditions}

A common set of boundary conditions that are used for clamped plates are the fixed-end conditions

$$
w(a, \theta)=\frac{\partial w}{\partial r}(a, \theta)=0
$$

$[10,13]$ in which it is assumed that zero displacements and slopes are maintained at the boundary of the plate. As noted in the introduction, however, it is impossible to maintain truly fixed boundary conditions in physical applications. This motivates the investigation of imperfectly clamped conditions which allow for rotations at the boundary as well as small transverse boundary displacements.

\subsection{Imperfectly Clamped Conditions}

When developing the imperfectly clamped conditions for the plate, we make the following assumptions:

1. The transverse boundary displacement and rotation are independent of $\theta$. In applications, this would be the case when clamping is not truly fixed but bolts are uniformly torqued along the boundary of the plate. Since a torque wrench is usually used to tighten the bolts, this is a reasonable assumption in many applications. In cases where uneven clamping along the boundary is suspected, analysis with $\theta$ dependent parameters can be used in a manner analogous to that presented here.

2. The bending moment $\mathcal{M}_{\theta}=M_{\theta}$ and twisting moment $\mathcal{M}_{r \theta}=M_{r \theta}$ are 0 at $r=a$. This assumption is based on the premise that, even with weakly clamped boundary conditions, the rotation at the boundary will be primarily in the radial direction.

To model the transverse motion at the clamped end, it is assumed that this boundary motion can be modeled using a spring-dashpot system on either side of the beam centerline as depicted in Figure 2.3. Here $\tilde{k}_{t}$ and $\tilde{c}_{t}$ are used to denote the equivalent spring coefficient and viscous damping coefficient, respectively. The balancing of forces then yields

$$
-\rho h \frac{\partial^{2} w}{\partial t^{2}}(t, a, \theta)=Q_{r}(t, a, \theta)+2 \tilde{k}_{t} w(t, a, \theta)+2 \tilde{c}_{t} \frac{\partial w}{\partial t}(t, a, \theta)
$$

Under the assumption that $\mathcal{M}_{\theta}=\mathcal{M}_{r \theta}=0$ at $r=a,(2.1)$ can be used to relate the shear resultant $Q_{r}$ to the moments through the expression

$$
Q_{r}=\frac{1}{a} M_{r}+\frac{\partial M_{r}}{\partial r}
$$




\section{MODEL FOR AN IMPERFECTLY CLAMPED PLATE}

(recall that $\mathcal{M}_{r}=M_{r}$ at the plate boundary). The displacement at $r=a$ then admits the differential equation

$$
\begin{aligned}
& \frac{1}{a} M_{r}(t, a, \theta)+\frac{\partial M_{r}}{\partial r}(t, a, \theta) \\
& \quad=-k_{t} w(t, a, \theta)-c_{t} \frac{\partial w}{\partial t}(t, a, \theta)-\rho h \frac{\partial^{2} w}{\partial t^{2}}(t, a, \theta) .
\end{aligned}
$$

The change in slope at the boundary is modeled by assuming that rotation at the clamp is constrained by a compressive moment with magnitude proportional to the tangent angle as depicted in Figure 2.4. Under the assumption of small rotations, $\tan \theta \approx \frac{\partial w}{\partial x}$ at $r=a$ which yields

$$
-M_{r}(t, a, \theta)=-k_{p} \frac{\partial w}{\partial r}(t, a, \theta)-c_{p} \frac{\partial^{2} w}{\partial r \partial t}(t, a, \theta) .
$$

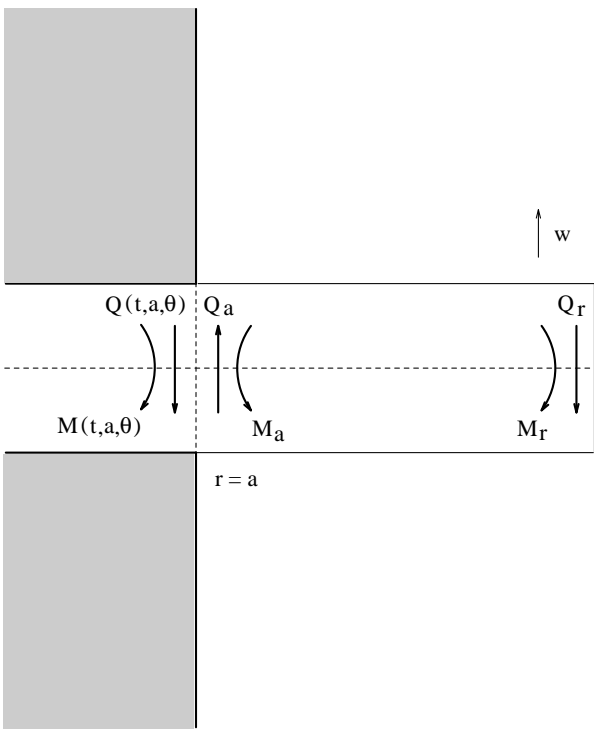

(a)

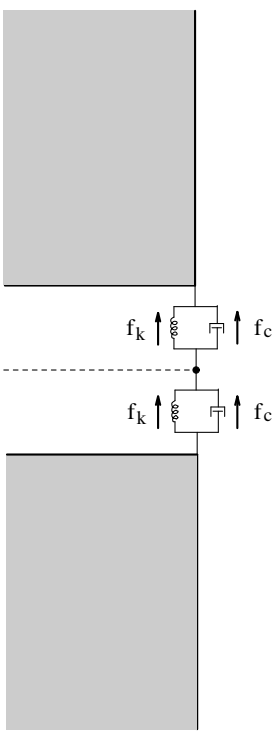

(b)

Figure 2.3: (a) Orientation of force and moment resultants. Note that $Q(t, a, \theta)=-Q_{a}$ and $M(t, a, \theta)=-M_{a}$. (b) Transverse boundary motion modeled as a spring-dashpot system. The forces are given by $f_{k}=\tilde{k}_{t}(t, a, \theta)$ and $f_{c}=\tilde{c}_{t} \frac{\partial u}{\partial t}(t, a, \theta)$. 


\section{H.T. BANKS, R.C. SMITH AND YUN WANG}
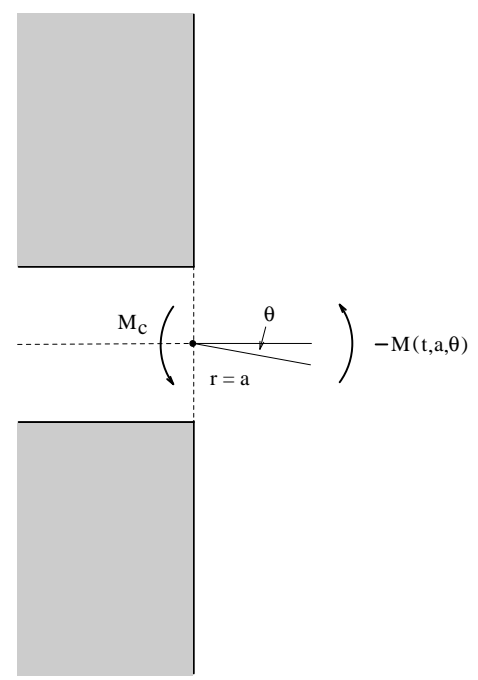

Figure 2.4: Boundary rotation and resulting moments.

\subsection{Strong Form of the Plate Equations}

The differential equation (2.3) and boundary conditions (2.8) and (2.9) can then be combined with initial conditions to yield the strong form of the circular plate equations

$$
\begin{aligned}
& \left\{\begin{array}{cc}
\rho h \frac{\partial^{2} w}{\partial t^{2}}-\frac{\partial^{2} \mathcal{M}_{r}}{\partial r^{2}}-\frac{2}{r} \frac{\partial \mathcal{M}_{r}}{\partial r}+\frac{1}{r} \frac{\partial \mathcal{M}_{\theta}}{\partial r}-\frac{2}{r} \frac{\partial^{2} \mathcal{M}_{r \theta}}{\partial r \partial \theta} & 0<\theta \leq 2 \pi
\end{array}-\frac{2}{r^{2}} \frac{\partial \mathcal{M}_{r \theta}}{\partial \theta}-\frac{1}{r^{2}} \frac{\partial^{2} \mathcal{M}_{\theta}}{\partial \theta^{2}}=f(t, r, \theta)\right. \\
& \left\{\begin{array}{c}
\frac{1}{a} M_{r}(t, a, \theta)+\frac{\partial M_{r}}{\partial r}(t, a, \theta) \\
=-k_{t} w(t, a, \theta)-c_{t} \frac{\partial w}{\partial t}(t, a, \theta)-\rho h \frac{\partial^{2} w}{\partial t^{2}}(t, a, \theta) \\
M_{r}(t, a, \theta)=k_{p} \frac{\partial w}{\partial r}(t, a, \theta)+c_{p} \frac{\partial^{2} w}{\partial r \partial t}(t, a, \theta)
\end{array}\right. \\
& \left\{\begin{array}{c}
w(0, r, \theta)=w_{0}(r, \theta) \quad, \quad \frac{\partial w}{\partial t}(0, r, \theta)=w_{1}(r, \theta) .
\end{array}\right.
\end{aligned}
$$

Explicit expressions for the moments are given in (2.4) and (2.5). It should be noted that since $k_{p}$ and $k_{t}$ are assumed to be constants, one can divide through by these quantities in the boundary conditions. In the limits 


\section{MODEL FOR AN IMPERFECTLY CLAMPED PLATE}

$k_{p} \rightarrow \infty$ and $k_{t} \rightarrow \infty$, the imperfectly clamped conditions converge to the fixed-end conditions (2.7). This should be expected since increased spring stiffnesses implies that one is approaching an ideal clamp in which displacements and slopes are truly zero.

\subsection{Weak Form of the Plate Equations}

To define a weak form of the equations of motion for the plate with weaklyclamped edges, we must determine an appropriate state, state space and space of test functions. The state is taken to be $z=(w(a, \cdot), w(\cdot, \cdot))$ in the space $H \equiv L^{2}(0,2 \pi) \times L^{2}\left(\Gamma_{0}\right)$. For $\Phi=(\zeta(\cdot), \phi(\cdot, \cdot))$ and $\Psi=(\xi(\cdot), \eta(\cdot, \cdot))$, the $H$-inner product is given by

$$
\langle\Phi, \Psi\rangle_{H}=\int_{0}^{2 \pi} \rho h a \zeta(\theta) \overline{\xi(\theta)} d \theta+\langle\rho h \phi, \eta\rangle
$$

where $\langle F, G\rangle \equiv \int_{\Gamma_{0}} F \bar{G} d \gamma$ with $d \gamma=r d \theta d r$. The overbar here denotes complex conjugation. To satisfy regularity requirements, the space of test functions and associated inner product are defined to be

$$
\begin{aligned}
V & \left.\equiv\{\Psi=(\xi(\cdot), \eta(\cdot, \cdot))) \in H: \eta \in H^{2}\left(\Gamma_{0}\right), \eta(a, \theta)=\xi(\theta)\right\} \\
\langle z, \Psi\rangle_{V} & =-\left\langle M_{r}, \frac{\partial^{2} \eta}{\partial r^{2}}\right\rangle-\left\langle\frac{1}{r} M_{\theta}, \frac{\partial \eta}{\partial r}\right\rangle-\left\langle\frac{1}{r^{2}} M_{\theta}, \frac{\partial^{2} \eta}{\partial \theta^{2}}\right\rangle \\
& -2\left\langle\frac{1}{r} M_{r \theta}, \frac{\partial^{2} \eta}{\partial r \partial \theta}\right\rangle+2\left\langle\frac{1}{r^{2}} M_{r \theta}, \frac{\partial \eta}{\partial \theta}\right\rangle \\
& +\int_{0}^{2 \pi} a k_{p} \frac{\partial w}{\partial r}(a, \theta) \frac{\overline{\partial \eta}}{\partial r}(a, \theta) d \theta+\int_{0}^{2 \pi} a k_{t} w(a, \theta) \bar{\eta}(a, \theta) d \theta
\end{aligned}
$$

where the moments are defined in (2.5) with $c_{D}=0$ in the respective definitions.

Integration of the internal moments in the second and third terms of (2.10) against the test functions yields

$$
\begin{aligned}
-\int_{\Gamma_{0}} & {\left[\frac{\partial^{2} M_{r}}{\partial r^{2}}+\frac{2}{r} \frac{\partial M_{r}}{\partial r}\right] \bar{\eta} d \gamma } \\
& =-\int_{\Gamma_{0}} M_{r} \frac{\partial^{2} \eta}{\partial r^{2}} d \gamma-\left.\int_{0}^{2 \pi}\left[\left(\frac{\partial M_{r}}{\partial r} r+M_{r}\right) \bar{\eta}-M_{r} r \frac{\overline{\partial \eta}}{\partial r}\right]\right|_{r=a} d \theta .
\end{aligned}
$$

In reducing the boundary term, we have used the fact that $M_{r}(t, 0, \theta)=0$ as a result of our choice of coordinate system. With the assumption that 


\section{H.T. BANKS, R.C. SMITH AND YUN WANG}

$M_{\theta}=M_{r \theta}=0$ at $r=a$, the remaining internal moment terms can be integrated by parts to yield

$$
\begin{aligned}
\int_{\Gamma_{0}}\left[\frac{1}{r} \frac{\partial M_{\theta}}{\partial r}-\frac{2}{r} \frac{\partial^{2} M_{r \theta}}{\partial r \partial \theta}-\frac{2}{r^{2}} \frac{\partial M_{r \theta}}{\partial \theta}-\frac{1}{r^{2}} \frac{\partial^{2} M_{\theta}}{\partial \theta^{2}}\right] \bar{\eta} d \gamma \\
\quad=-\int_{\Gamma_{0}} \frac{1}{r^{2}} M_{\theta}\left[r \frac{\overline{\partial \eta}}{\partial r}+\overline{\frac{\partial^{2} \eta}{\partial \theta^{2}}}\right] d \gamma-2 \int_{\Gamma_{0}} \frac{1}{r^{2}} M_{r \theta}\left[r \overline{\frac{\partial^{2} \eta}{\partial r \partial \theta}}-\overline{\frac{\partial \eta}{\partial \theta}}\right] d \gamma
\end{aligned}
$$

for all $\Psi \in V$. Inclusion of external moments and forces and consolidation of terms then gives the weak form

$$
\begin{aligned}
& \int_{\Gamma_{0}} \rho h \frac{\partial^{2} w}{\partial t^{2}} \bar{\eta} d \gamma-\int_{\Gamma_{0}} M_{r} \frac{\overline{\partial^{2} \eta}}{\partial r^{2}} d \gamma-\int_{\Gamma_{0}} \frac{1}{r^{2}} M_{\theta}\left[r \overline{\frac{\partial \eta}{\partial r}}+\overline{\frac{\partial^{2} \eta}{\partial \theta^{2}}}\right] d \gamma \\
& -2 \int_{\Gamma_{0}} \frac{1}{r^{2}} M_{r \theta}\left[r \frac{\partial^{2} \eta}{\partial r \partial \theta}-\frac{\overline{\partial \eta}}{\partial \theta}\right] d \gamma \\
& +\int_{0}^{2 \pi} a\left[k_{t} w(t, a, \theta)+c_{t} \frac{\partial w}{\partial t}(t, a, \theta)+\rho h \frac{\partial^{2} w}{\partial t^{2}}(t, a, \theta)\right] \bar{\eta}(a, \theta) d \theta \\
& +\int_{0}^{2 \pi} a\left[k_{p} \frac{\partial w}{\partial r}(t, a, \theta)+c_{p} \frac{\partial^{2} w}{\partial r \partial t}(t, a, \theta)\right] \frac{\overline{\partial \eta}}{\partial r}(a, \theta) d \theta \\
& =-\int_{\Gamma_{0}} \sum_{i=1}^{s} \mathcal{K}_{i}^{B} u_{i}(t) \chi_{i}(r, \theta) \overline{\nabla^{2} \eta} d \gamma+\int_{\Gamma_{0}} f \bar{\eta} d \gamma
\end{aligned}
$$

for all $\Psi=(\eta(a, \cdot), \eta(\cdot, \cdot)) \in V$. Again, explicit expressions for the internal moments are given in (2.5). It is easily noted that in this form, derivatives that were originally applied to moments have been transferred onto the test functions. This eliminates the difficulties associated with differentiating the piecewise constant parameters $D, \nu$ and $c_{D}$ found in the internal moments as well as the discontinuous input parameters $\mathcal{K}_{i}^{B}$.

\section{Model Well-Posedness}

A first step in proving the existence of a unique solution to these modeling equations is to pose the problem in abstract first-order form. To do so, we 


\section{MODEL FOR AN IMPERFECTLY CLAMPED PLATE}

first define the sesquilinear forms $\sigma_{i}: V \times V \rightarrow \mathbb{C}, i=1,2$ by

$$
\begin{aligned}
\sigma_{1}(W, \Psi) & =\int_{\Gamma_{0}} D\left(\frac{\partial^{2} w}{\partial r^{2}}+\frac{\nu}{r} \frac{\partial w}{\partial r}+\frac{\nu}{r^{2}} \frac{\partial^{2} w}{\partial \theta^{2}}\right) \frac{\overline{\partial^{2} \eta}}{\partial r^{2}} d \gamma \\
& +\int_{\Gamma_{0}} D\left(\frac{1}{r^{2}} \frac{\partial w}{\partial r}+\frac{1}{r^{3}} \frac{\partial^{2} w}{\partial \theta^{2}}+\frac{\nu}{r} \frac{\partial^{2} w}{\partial r^{2}}\right) \overline{\frac{\partial \eta}{\partial r}} d \gamma \\
& +\int_{\Gamma_{0}} D\left(\frac{1}{r^{3}} \frac{\partial w}{\partial r}+\frac{1}{r^{4}} \frac{\partial^{2} w}{\partial \theta^{2}}+\frac{\nu}{r^{2}} \frac{\partial^{2} w}{\partial r^{2}}\right) \overline{\frac{\partial^{2} \eta}{\partial \theta^{2}}} d \gamma \\
& +\int_{\Gamma_{0}} 2(1-\nu) D\left(\frac{1}{r^{2}} \frac{\partial^{2} w}{\partial \theta \partial r}-\frac{1}{r^{3}} \frac{\partial w}{\partial \theta}\right) \frac{\partial^{2} \eta}{\partial r \partial \theta} d \gamma \\
& +\int_{\Gamma_{0}} 2(1-\nu) D\left(-\frac{1}{r^{3}} \frac{\partial^{2} w}{\partial \theta \partial r}+\frac{1}{r^{4}} \frac{\partial w}{\partial \theta}\right) \overline{\frac{\partial \eta}{\partial \theta}} d \gamma \\
& +\int_{0}^{2 \pi} a k_{p} \frac{\partial w}{\partial r}(a, \theta) \frac{\partial \eta}{\partial r}(a, \theta) d \theta+\int_{0}^{2 \pi} a k_{t} w(a, \theta) \bar{\eta}(a, \theta) d \theta
\end{aligned}
$$

and

$$
\begin{aligned}
\sigma_{2}(W, \Psi) & =\int_{\Gamma_{0}} c_{D}\left(\frac{\partial^{2} w}{\partial r^{2}}+\frac{\nu}{r} \frac{\partial w}{\partial r}+\frac{\nu}{r^{2}} \frac{\partial^{2} w}{\partial \theta^{2}}\right) \overline{\frac{\partial^{2} \eta}{\partial r^{2}}} d \gamma \\
& +\int_{\Gamma_{0}} c_{D}\left(\frac{1}{r^{2}} \frac{\partial w}{\partial r}+\frac{1}{r^{3}} \frac{\partial^{2} w}{\partial \theta^{2}}+\frac{\nu}{r} \frac{\partial^{2} w}{\partial r^{2}}\right) \overline{\frac{\partial \eta}{\partial r}} d \gamma \\
& +\int_{\Gamma_{0}} c_{D}\left(\frac{1}{r^{3}} \frac{\partial w}{\partial r}+\frac{1}{r^{4}} \frac{\partial^{2} w}{\partial \theta^{2}}+\frac{\nu}{r^{2}} \frac{\partial^{2} w}{\partial r^{2}}\right) \overline{\frac{\partial^{2} \eta}{\partial \theta^{2}}} d \gamma \\
& +\int_{\Gamma_{0}} 2(1-\nu) c_{D}\left(\frac{1}{r^{2}} \frac{\partial^{2} w}{\partial \theta \partial r}-\frac{1}{r^{3}} \frac{\partial w}{\partial \theta}\right) \frac{\partial^{2} \eta}{\partial r \partial \theta} d \gamma \\
& +\int_{\Gamma_{0}} 2(1-\nu) c_{D}\left(-\frac{1}{r^{3}} \frac{\partial^{2} w}{\partial \theta \partial r}+\frac{1}{r^{4}} \frac{\partial w}{\partial \theta}\right) \frac{\overline{\partial \eta}}{\partial \theta} d \gamma \\
& +\int_{0}^{2 \pi} a c_{p} \frac{\partial w}{\partial r}(a, \theta) \frac{\partial \eta}{\partial r}(a, \theta) d \theta+\int_{0}^{2 \pi} a c_{t} w(a, \theta) \bar{\eta}(a, \theta) d \theta
\end{aligned}
$$

With $\langle\cdot, \cdot\rangle_{V^{*}, V}$ denoting the usual duality product, $F=\left(0, \frac{f}{p h}\right)$ and $z(t)=(w(t, a, \cdot), w(t, \cdot, \cdot))$, the system (2.11) containing the weak form of the modeling equations can then be written as

$$
\left\langle z_{t t}(t), \Psi\right\rangle_{V^{*}, V}+\sigma_{2}\left(z_{t}(t), \Psi\right)+\sigma_{1}(z(t), \Psi)=\langle F+B, \Psi\rangle_{V^{*}, V}
$$




\section{H.T. BANKS, R.C. SMITH AND YUN WANG}

for $\Psi$ in $V$. The input operator $B \in \mathcal{L}\left(\mathrm{IR}^{s}, V^{*}\right)$ defined by

$$
\langle B u, \Psi\rangle_{V^{*}, V}=-\int_{\Gamma_{0}} \sum_{i=1}^{s} \mathcal{K}_{i}^{B} u_{i} \chi_{i}(r, \theta) \overline{\nabla^{2} \eta} d \gamma
$$

contains the contributions due to the excitation of the patches through applied voltages.

Before writing the system in first-order form and proving well-posedness, we must further examine the properties of the sesquilinear forms. It is first noted that $\sigma_{1}$ satisfies

$$
\begin{array}{lll}
\left|\sigma_{1}(\Phi, \Psi)\right| \leq c_{1}|\Phi|_{V}|\Psi|_{V} & \text {, for some } c_{1} \in \mathbb{R} & \text { (bounded) } \\
\operatorname{Re} \sigma_{1}(\Phi, \Phi) \geq c_{2}|\Phi|_{V}^{2} & \text {, for some } c_{2}>0 & \text { (V-elliptic) } \\
\sigma_{1}(\Phi, \Psi)=\overline{\sigma_{1}(\Psi, \Phi)} & \text { (symmetric) }
\end{array}
$$

for all $\Phi, \Psi \in V$. The boundedness follows from Schwarz's inequality for inner products in conjunction with equivalence results for various Sobolev norms. The $V$-ellipticity and symmetry of $\sigma_{1}$ follow directly from the fact that $\sigma_{1}(\Phi, \Psi)=\langle\Phi, \Psi\rangle_{V}$.

Under the assumption that the damping parameters satisfy $c_{D}(r, \theta) \geq$ $\overline{c_{D}}>0, c_{p}>0$ and $c_{t}>0$, it also follows that

$$
\begin{array}{lll}
\left|\sigma_{2}(\Phi, \Psi)\right| \leq c_{3}|\Phi|_{V}|\Psi|_{V} & \text {, for some } c_{3} \in \mathbb{R} & \text { (bounded) } \\
\operatorname{Re} \sigma_{2}(\Phi, \Phi) \geq c_{4}|\Phi|_{V}^{2} & \text {, for some } c_{4}>0 & \text { (V-elliptic) }
\end{array}
$$

To prove convergence in the parameter estimation problem, we also need to show continuity of the sesquilinear forms with respect to parameters. To this end, we let

$$
q=\left(\rho, D, \nu, c_{D}, k_{t}, k_{p}, c_{t}, c_{p}, \mathcal{K}_{1}^{B}, \cdots, \mathcal{K}_{s}^{B}\right)
$$

and let $d$ denote the standard metric in the metric space $\tilde{Q}=\left[L_{\infty}\left(\Gamma_{0}\right)\right]^{4} \times$ $\mathbb{R}^{4} \times \mathbb{R}^{s}$ (details regarding this choice of metric space will be given in the Section 5). Using Schwarz's inequality for inner products in conjunction with appropriate Sobolev inequalities, it can then be shown that for $q, \tilde{q} \in \tilde{Q}$ and $\Psi, \Phi \in V$,

$$
\begin{aligned}
& \left|\sigma_{1}(q)(\Phi, \Psi)-\sigma_{1}(\tilde{q})(\Phi, \Psi)\right| \leq \gamma_{1} d(q, \tilde{q})|\Phi|_{V}|\Psi|_{V} \\
& \left|\sigma_{2}(q)(\Phi, \Psi)-\sigma_{2}(\tilde{q})(\Phi, \Psi)\right| \leq \gamma_{2} d(q, \tilde{q})|\Phi|_{V}|\Psi|_{V}
\end{aligned}
$$

Hence both sesquilinear forms are continuous with respect to parameters as well as satisfy the previously discussed continuity, coercivity and symmetry conditions. 


\section{MODEL FOR AN IMPERFECTLY CLAMPED PLATE}

To pose the system in first-order form, we form the product space terms $\mathcal{B} u(t)=(0, B u(t))$ and $\mathcal{F}(t)=(0, F(t))$ in $\mathcal{V}^{*}=V \times V^{*}$ and define the operators $A_{1}, A_{2} \in \mathcal{L}\left(V, V^{*}\right)$ by

$$
\left\langle A_{i} \Phi, \Psi\right\rangle_{V^{*}, V}=\sigma_{i}(\Phi, \Psi)
$$

for $i=1,2$ (the existence of $A_{1}$ and $A_{2}$ is guaranteed by the boundedness of $\sigma_{1}$ and $\left.\sigma_{2}\right)$. Then, for the state $\mathcal{Z}(t)=(z, \dot{z})$ in $\mathcal{H}=H \times V$, the weak form (3.1) is formally equivalent to the system

$$
\dot{\mathcal{Z}}(t)=\mathcal{A} \mathcal{Z}(t)+\mathcal{B} u(t)+\mathcal{F}(t)
$$

in $\mathcal{V}^{*}$ where

$$
\begin{gathered}
\operatorname{dom} \mathcal{A}=\left\{\Theta=(\Upsilon, \Lambda) \in \mathcal{H}: \Lambda \in V, A_{1} \Upsilon+A_{2} \Lambda \in H\right\} \\
\mathcal{A}=\left[\begin{array}{cc}
0 & I \\
-A_{1} & -A_{2}
\end{array}\right] .
\end{gathered}
$$

(the representation is formal in the sense that the manner in which differentiation and the resulting solution exist has not yet been specified).

To argue the well-posedness of the model, it is first noted that the sesquilinear forms $\sigma_{1}$ and $\sigma_{2}$ are V-elliptic and continuous and $\sigma_{1}$ is symmetric. From the Lumer-Philips theorem (with further arguments found on pages 82-84 of [1]) this then implies that the operator $\mathcal{A}$ defined in (3.6) generates an analytic semigroup $\mathcal{T}$ on the state space $\mathcal{H}$. Moreover, the semigroup satisfies the exponential bound $|\mathcal{T}(t)| \leq e^{\omega t}$ for $t \geq 0$ (where in fact, $\omega=0$ due to the fact that $\mathcal{A}$ is dissipative as shown in [1]).

In the case of bounded input operators, one would then define a mild solution on $\mathcal{H}$ and show that this mild solution is indeed a strong solution to the problem. Here, however, $\mathcal{B} u(t)$ lies in $\mathcal{V}^{*}$ rather than $\mathcal{H}$ and one must extend the semigroup $\mathcal{T}(t)$ on $\mathcal{H}$ to a semigroup $\tilde{\mathcal{T}}(t)$ on a larger space $\mathcal{W}^{*} \supset\{0\} \times V^{*}$ so as to be compatible with the forcing term.

As detailed in $[3,6]$, the space of interest is defined in terms of $\operatorname{dom} \mathcal{A}^{*}$ where

$$
\begin{gathered}
\operatorname{dom} \mathcal{A}^{*}=\left\{\chi=(\Phi, \Psi) \in \mathcal{H} \mid \Psi \in V, A_{1}^{*} \Phi-A_{2}^{*} \Psi \in H\right\} \\
\mathcal{A}^{*} \chi=\left(\begin{array}{c}
-\Psi \\
A_{1}^{*} \Phi-A_{2}^{*} \Psi
\end{array}\right) .
\end{gathered}
$$

Specifically, the space $\mathcal{W}=\left[\operatorname{dom} \mathcal{A}^{*}\right]$ is taken to be $\operatorname{dom} \mathcal{A}^{*}$ with the inner product

$$
\langle\Phi, \Psi\rangle_{\mathcal{W}}=\left\langle\left(\lambda_{0}-\mathcal{A}^{*}\right) \Phi,\left(\lambda_{0}-\mathcal{A}^{*}\right) \Psi\right\rangle_{\mathcal{H}}
$$




\section{H.T. BANKS, R.C. SMITH AND YUN WANG}

for some arbitrary but fixed $\lambda_{0}$ with $\lambda_{0}>\omega$ (recall that the original solution semigroup satisfies the bound $\left.|\mathcal{T}(t)| \leq e^{\omega t}\right)$. By employing "extrapolation space" ideas and arguments similar to those presented in [3, 9], one can then extend the operator $\mathcal{A}: \operatorname{dom} \mathcal{A} \subset \mathcal{H} \rightarrow \mathcal{H}$ to an operator $\tilde{\mathcal{A}}$ which is defined on all of $\mathcal{H}$. Moreover, the operator $\tilde{\mathcal{A}}$ is the infinitesimal generator of an analytic semigroup $\tilde{\mathcal{T}}(t)$ which is an extension of $\mathcal{T}$ from $\mathcal{H}$ to $\mathcal{W}^{*}$.

Using this extension, the following theorem from [3] then guarantees the existence of a unique solution as well verifies the equivalence of the strong and weak solutions.

Theorem 3.1: Existence and Equivalence of Solutions) Consider the system represented by (2.11) or (3.1) and suppose that the mappings $t \mapsto\left\{u_{i}(t)\right\}$ and $t \mapsto F(t)$ from $[0, T]$ to $\mathbb{R}^{s}$ and $V^{*}$, respectively, are Lipschitz continuous. Then for each $\mathcal{Z}_{0} \in \mathcal{H}=\operatorname{dom} \tilde{\mathcal{A}}$, we have that (3.5) taken with $\mathcal{Z}(0)=\mathcal{Z}_{0}$ has a unique strong solution given by

$$
\mathcal{Z}(t)=\tilde{\mathcal{T}}(t) \mathcal{Z}_{0}+\int_{0}^{t} \tilde{\mathcal{T}}(t-s)\left(\begin{array}{c}
0 \\
B u(s)+F(s)
\end{array}\right) d s
$$

Moreover, this strong solution is equal to the weak solution of (2.11) or (3.1).

As a result of this theorem, the imperfectly clamped plate model is wellposed. We turn next to the issue of estimating parameters in this model.

\section{State Approximation}

Having established the existence of a solution to the imperfectly clamped plate model, we turn now to the issue of approximating the infinite dimensional system dynamics. As discussed in [5, 12], an appropriate choice for the basis and Fourier-Galerkin expansion of the plate displacement is $B_{k}^{\mathcal{N}}(r, \theta)=r^{|\hat{m}|} B_{n}^{m}(r) e^{i m \theta}$ and

$$
\begin{aligned}
w^{\mathcal{N}}(t, r, \theta) & =\sum_{m=-M}^{M} \sum_{n=1}^{N^{m}} w_{m n}^{\mathcal{N}}(t) r^{|\hat{m}|} B_{n}^{m}(r) e^{i m \theta} \\
& =\sum_{k=1}^{\mathcal{N}} w_{k}^{\mathcal{N}}(t) B_{k}^{\mathcal{N}}(r, \theta) .
\end{aligned}
$$

In the previously cited references, basis alterations were needed to accommodate the displacement and slope conditions mandated by the fixed boundary conditions. This is not necessary when considering imperfectly 


\section{MODEL FOR AN IMPERFECTLY CLAMPED PLATE}

clamped boundary conditions since it is no longer required that the approximate solution satisfy $w^{\mathcal{N}}(t, a, \theta)=\frac{\partial w^{\mathcal{N}}}{\partial r}(t, a, \theta)=0$. In this case, $B_{n}^{m}(r)$ denotes a cubic spline that is modified so as to satisfy the condition $\frac{d B_{n}^{m}(0)}{d r}=0$ when $m=0$. This guarantees differentiability at the origin and implies that

$$
N^{m}= \begin{cases}N+3 & , \quad m=0 \\ N+2, & m \neq 0\end{cases}
$$

where $N$ denotes the number of modified cubic splines. Thus a total of $\mathcal{N}=(2 M+1)(N+3)-1$ basis functions are used when approximating the plate displacement. As discussed in the [5, 12], the inclusion of the weighting term $r^{|\hat{m}|}$ with

$$
\hat{m}= \begin{cases}0, & m=0 \\ 1, & m \neq 0\end{cases}
$$

is motivated by the asymptotic behavior of the Bessel functions (which make up the analytic plate solution) as $r \rightarrow 0$. It also serves to ensure the uniqueness of the solution at the origin. The Fourier coefficient in the weight is truncated to control the conditioning of the mass and stiffness matrices (see the examples in [5]).

To obtain a matrix system, the $\mathcal{N}$ dimensional approximating subspace is taken to be $H^{\mathcal{N}}=\operatorname{span}\left\{B_{k}^{\mathcal{N}}\right\}$ and the product space for the first-order system is $\mathcal{H}^{\mathcal{N}} \times \mathcal{H}^{\mathcal{N}}$. The restriction of the infinite-dimensional system (2.11), or equivalently (3.1), to the space $\mathcal{H}^{\mathcal{N}} \times \mathcal{H}^{\mathcal{N}}$ then yields a matrix system of the form

$$
\begin{aligned}
& \dot{y}^{\mathcal{N}}(t)=A^{\mathcal{N}} y^{\mathcal{N}}(t)+B^{\mathcal{N}} u(t)+F^{\mathcal{N}}(t) \\
& y^{\mathcal{N}}(0)=y_{0}^{\mathcal{N}}
\end{aligned}
$$

where $y^{\mathcal{N}}(t)=\left[w_{1}(t), \cdots, w_{N}(t), \dot{w}_{1}(t), \cdots, \dot{w}_{N}(t)\right]$ denotes the $2 \mathcal{N} \times 1$ vector containing the generalized Fourier coefficients for the approximate displacement and velocity. Details concerning the construction of the component vectors and matrices in $(4.2)$ can be found in [5, 12].

\section{Parameter Estimation}

We now turn to the problem of estimating the physical plate parameters $\rho, D, \nu, c_{D}, k_{t}, k_{p}, c_{t}, c_{p}, \mathcal{K}_{1}^{B}, \cdots, \mathcal{K}_{s}^{B}$ by fitting data $z$ obtained from displacement, velocity or acceleration measurements at various locations on the plate. To formulate the problem in an optimization setting, we let $q=\left(\rho, D, \nu, c_{D}, k_{t}, k_{p}, c_{t}, c_{p}, \mathcal{K}_{1}^{B}, \cdots, \mathcal{K}_{s}^{B}\right)$ and assume that $q \in Q$ where 


\section{H.T. BANKS, R.C. SMITH AND YUN WANG}

the admissible parameter space $Q$ is taken to be a compact subset of the metric space $\tilde{Q}=\left[L_{\infty}\left(\Gamma_{0}\right)\right]^{4} \times \mathbb{R}^{4} \times \mathbb{R}^{s}$ with elements also satisfying the physical constraints $\rho>0, D>0, \nu>0$ and $c_{D}>0$ on $\Gamma_{0}$. Associated with the space $\tilde{Q}$ is the standard metric $d$.

For time domain estimation with data consisting of position, velocity, or acceleration measurements at points $(\hat{r}, \hat{\theta})$ on the plate, the infinite dimensional parameter estimation problem is to seek $\bar{q}$ which minimizes

$$
J(q)=\sum_{i}\left|\frac{\partial^{\nu} w}{\partial t^{\nu}}\left(t_{i}, \hat{r}, \hat{\theta} ; q\right)-z_{i}\right|^{2} \quad, \quad \nu=0,1 \text { or } 2
$$

subject to $w$ satisfying (2.11) or (3.1). As noted in [8], similar penalty functionals can be used if the data consists of accumulated strain measurements obtained from the patches.

To facilitate the estimation of the material parameters, we now make some assumptions regarding their spatial behavior. As discussed previously, the parameters $\rho, D, \nu$ and $c_{D}$ are assumed to be piecewise constants in order to account for the presence and differing material properties of the piezoceramic patches. For the case in which $s$ patches or patch pairs are bonded to the plate, these parameters can then be expressed as

$$
\begin{aligned}
\rho(r, \theta) & =\sum_{i=1}^{s+1} c_{\rho i} \chi_{i}(r, \theta) \quad, & D(r, \theta) & =\sum_{i=1}^{s+1} c_{D i} \chi_{i}(r, \theta) \\
\nu(r, \theta) & =\sum_{i=1}^{s+1} c_{\nu i} \chi_{i}(r, \theta) \quad, & c_{D}(r, \theta) & =\sum_{i=1}^{s+1} c_{c_{D} i} \chi_{i}(r, \theta)
\end{aligned}
$$

where again, $\chi_{i}(r, \theta), i=1, \cdots, s$ is the characteristic functions over the $i^{t h}$ patch or patch pair and $\chi_{s+1}$ is the characteristic function over the portion of the plate not covered with patches. The parameters $k_{t}, k_{p}, c_{t}$ and $c_{p}$ are constants which must also be estimated when using this plate model. Finally, we recall from the definition (2.6) that the patch parameters $\mathcal{K}_{1}^{B}, \cdots, \mathcal{K}_{s}^{B}$ are constants which depend on piezoelectric properties, the geometry and size of the patch, and bonding layer and patch properties.

To provide an optimization problem involving a finite dimensional state, the approximation techniques discussed in the previous section are employed. The finite dimensional minimization problem corresponding to (5.1) is to then seek $\bar{q} \in Q$ which minimizes

$$
J^{N}(q)=\sum_{i}\left|\frac{\partial^{\nu} w^{\mathcal{N}}}{\partial t^{\nu}}\left(t_{i}, \hat{r}, \hat{\theta} ; q\right)-z_{i}\right|^{2} \quad, \quad \nu=0,1,2
$$

subject to $w^{\mathcal{N}}$ satisfying the approximate plate equations (hence the coefficients $\left\{w_{k}^{\mathcal{N}}\right\}$ of $w^{\mathcal{N}}$ must satisfy $(4.2)$. 


\section{MODEL FOR AN IMPERFECTLY CLAMPED PLATE}

The following theorem, taken from [8], specifies conditions under which convergence and continuous dependence (on data) of the solutions to the finite dimensional parameter estimation problems involving the functional (5.2) can be expected.

Theorem 5.1 Let $Q$ be a compact subset of a metric space $\tilde{Q}$ with metric $d$ and assume that $H^{N} \subset V$ approximates $V$ in the sense that for each $\Phi \in V$, there exists $\Phi^{N} \in H^{N}$ such that

$$
\left|\Phi-\Phi^{N}\right|_{V} \leq \varepsilon(N) \rightarrow 0 \text { as } N \rightarrow \infty .
$$

Furthermore, assume that $\sigma_{1}(q)$ and $\sigma_{2}(q)$ are $V$-elliptic, continuous, and satisfy the continuity with respect to parameter condition

$$
\left|\sigma_{i}(q)(\Phi, \Psi)-\sigma_{i}(\tilde{q})(\Phi, \Psi)\right| \leq \gamma_{i} d(q, \tilde{q})|\Phi|_{V}|\Psi|_{V} \quad \text {, for } \Phi, \Psi \in V
$$

for $i=1,2$ and $q, \tilde{q} \in Q$. Finally, assume that

$$
q \mapsto(B u+F)(t ; q) \text { is continuous from } Q \text { to } L^{2}\left((0, T), V^{*}\right) .
$$

For arbitrary $q^{N}$ such that $q^{N} \rightarrow q$ in $Q$, one then has the convergence

$$
\begin{aligned}
& z^{N}\left(t ; q^{N}\right) \rightarrow z(t ; q) \quad \text { in } V \text { norm } \\
& z_{t}^{N}\left(t ; q^{N}\right) \rightarrow z_{t}(t ; q) \quad \text { in } V \text { norm }
\end{aligned}
$$

for $t>0$. Here $z$ and $z_{t}$ are solutions to the system (2.11) or (3.1) and $z^{N}$ and $z_{t}^{N}$ solve the corresponding linear finite dimensional system in $H^{N}$.

In considering the hypotheses, we note that the sesquilinear terms satisfy the ellipticity and continuity conditions due to the assumption of KelvinVoigt damping in the plate model. As noted in (3.4), the sesquilinear terms also satisfy the parameter continuity condition. Moreover, the input term $(F+B u)(q)$ satisfies the condition (5.5). Finally, the convergence condition specified in (5.3) is satisfied as a consequence of the approximating properties of the cubic splines and Fourier elements.

For $\nu=0,1$, it then follows directly that there exists a subsequence of solutions $\bar{q}^{N}$ minimizing (5.2) subject to (4.2) which converge to a solution $\bar{q}$ of the original minimization problem (5.1) subject to (2.11) or (3.1). The convergence in the case involving the minimization of (5.2) with acceleration data does not follow directly from this theorem but can be obtained using results from the theory of analytic semigroups in a manner analogous to that used in [4]. 


\section{H.T. BANKS, R.C. SMITH AND YUN WANG}

\section{Conclusions}

In this paper, Newtonian principles were used to derive a model for boundary conditions for an imperfectly clamped thin circular plate. Under the assumption that some boundary displacement and rotation occur during oscillations, boundary moments, involving parameters to be estimated through fit-to-data techniques, were included in the modeling equations for the plate. It was demonstrated that these boundary conditions converge to fixed-end boundary conditions (zero displacement and slope) as the parameters modeling boundary stiffness tend to infinity.

An equivalent weak form of the modeling equations was then derived. By formulating this weak form in terms of sesquilinear forms and corresponding bounded operators, a semigroup formulation of the problem was developed. Extrapolation techniques were used to extend the semigroup to a space compatible with the input terms at which point, well-posedness of the system model and equivalence between strong and weak solutions were demonstrated. Finally, techniques for approximating the plate dynamics were outlined and criteria leading to parameter convergence when estimating parameters (including parameters in the boundary conditions) were discussed.

This provides a physical model which can be used when investigating the mechanism for energy loss through boundary supports. It also provides a means of testing the suitability of fixed-end boundary conditions in applications in which the structure is securely clamped.

\section{Acknowledgements}

The authors would like to thank Rich Silcox and Jay Robinson, Acoustics Division, NASA Langley Research Center, for input and insights provided during discussions regarding the effects of weakly clamped boundary conditions on experimental plate data. We also thank Ken Bowers, John Lund and the staff in the Department of Mathematical Sciences at Montana State University for the organization and assistance provided during this conference.

\section{References}

[1] H.T. BANKS and K. ITO, "A Unified Framework for Approximation in Inverse Problems for Distributed Parameter Systems," ControlTheory and Advanced Technology v. 4, no. 1, 1988, pp. 73-90. 


\section{MODEL FOR AN IMPERFECTLY CLAMPED PLATE}

[2] H.T. BANKS and K. ITO, "Approximation in LQR Problems for Infinite Dimensional Systems with Unbounded Input Operators," submitted to Journal of Mathematical Systems, Estimation and Control.

[3] H.T. BANKS, K. ITO and Y. WANG, "Well-Posedness for Damped Second Order Systems with Unbounded Input Operators," Center for Research in Scientific Computation Technical Report, CRSC-TR9310, North Carolina State University, to appear in Differential and Integral Equations, March 1995.

[4] H.T. BANKS and D.A. REBNORD, "Analytic Semigroups: Applications to Inverse Problems for Flexible Structures," Differential Equations with Applications, (ed. by J. Goldstein, et. al.), Marcel Dekker, 1991, pp. 21-35.

[5] H.T. BANKS and R.C. SMITH, "The Modeling and Approximation of a Structural Acoustics Problem in a Hard-Walled Cylindrical Domain," to appear as a Center for Research in Scientific Computation Technical Report.

[6] H.T. BANKS and R.C.SMITH, "Well-Posedness of a Model for Structural Acoustic Coupling in a Cavity Enclosed by a Thin Cylindrical Shell," ICASE Report 93-10, to appear in Journal of Mathematical Analysis and Applications.

[7] H.T. BANKS, R.C. SMITH and Y. WANG, "Modeling Aspects for Piezoceramic Patch Activation of Shells, Plates and Beams," Center for Research in Scientific Computation Technical Report, CRSCTR92-12, N. C. State Univ., to appear in Quarterly of Applied Mathematics.

[8] H.T. BANKS, Y. WANG, D.J. INMAN and J.C. SLATER, "Approximation and Parameter Identification for Damped Second Order Systems with Unbounded Input Operators," Center for Research in Scientific Computation Technical Report, CRSC-TR93-9, North Carolina State University, to appear in Control: Theory and Advanced Technology.

[9] A. HARAUX, "Linear Semigroups in Banach Spaces," in Semigroups, Theory and Applications, II (H. Brezis, et al., eds.), Pitman Res. Notes in Math, Vol 152, Longman, London, 1986, pp. 93-135. 


\section{H.T. BANKS, R.C. SMITH AND YUN WANG}

[10] E.H. MANSFIELD, The Bending and Stretching of Plates, Volume 6 in the International Series of Monographs on Aeronautics and Astronautics, The MacMillan Company, New York, 1964.

[11] J. ROBINSON, Acoustics Division, NASA Langley Research Center, personal communications.

[12] R.C. SMITH, "A Galerkin Method for Linear PDE Systems in Circular Geometries with Structural Acoustic Applications," ICASE Report No. 94-40, submitted to SIAM Journal on Scientific Computing.

[13] W. SOEDEL, Vibrations of Shells and Plates, Second Edition, Marcel Dekker, Inc., New York, 1993.

[14] S. TIMOSHENKO, Theory of Plates and Shells, McGraw-Hill, NY, 1940. 